\title{
FAST QUATERNION ATTITUDE ESTIMATION FROM TWO VECTOR MEASUREMENTS
}

\author{
F. Landis Markley \\ Guidance, Navigation, and Control Systems Engineering Branch, Code 571 \\ NASA's Goddard Space Flight Center, Greenbelt, MD 2077 I
}

\begin{abstract}
Many spacecraft attitude determination methods use exactly two vector measurements. The two vectors are typically the unit vector to the Sun and the Earth's magnetic field vector for coarse "sun-mag" attitude determination or unit vectors to two stars tracked by two star trackers for fine attitude determination. Existing closed-form attitude estimates based on Wahba's optimality criterion for two arbitrarily weighted observations are somewhat slow to evaluate. This paper presents two new fast quaternion attitude estimation algorithms using two vector observations, one optimal and one suboptimal. The suboptimal method gives the same estimate as the TRIAD algorithm, at reduced computational cost. Simulations show that the TRIAD estimate is almost as accurate as the optimal estimate in representative test scenarios.
\end{abstract}

\section{INTRODUCTION}

Suppose that we have measured two unit vectors $b_{1}$ and $b_{2}$ in the spacecraft body frame, e.g. unit vectors along the line of sight to a star or the Sun, or along the Earth's magnetic field. We consider only unit vectors because the length of the vector has no information directly relevant to attitude determination. Each of these unit vectors contains two independent scalar pieces of attitude information. The spacecraft attitude is represented by a $3 \times 3$ proper orthogonal matrix $A$, i.e. $A^{T} A=I$, the $3 \times 3$ identity matrix, and $\operatorname{det} A=1$. Since $A$ is thus an element of the three-parameter group $\operatorname{SO}(3)$, two unit vector measurements contain one more piece of information than is necessary to determine the attitude matrix.

It is also necessary to know the components of the two measured vectors $\boldsymbol{r}_{1}$ and $\mathbf{r}_{2}$ in some reference frame. The reference frame is usually taken to be an inertial frame, but this is not necessary. One can use a rotating frame such as the frame referenced to the orbit normal vector and the local vertical. The attitude matrix to be determined is the matrix that rotates vectors from the reference frame to the spacecraft body frame. Thus we would like to find an attitude matrix such that

$$
A \mathbf{r}_{1}=\mathbf{b}_{1}
$$

and

$$
A \mathbf{r}_{2}=\mathbf{b}_{2} \text {. }
$$

This is not possible in general, however, for Eq. (1) implies that

$$
\mathbf{b}_{1} \cdot \mathbf{b}_{2}=\left(A \mathbf{r}_{1}\right) \cdot\left(A \mathbf{r}_{2}\right)=\mathbf{r}_{1} \cdot \mathbf{r}_{2}
$$

This equality is true for error-free measurements, but is not generally true in the presence of measurement errors. All reasonable two-vector attitude determination schemes give the same estimate when Eq. (2) is valid.

It is clear from simple counting arguments that the two independent scalar pieces of information contained in a single vector measurement cannot determine the attitude uniquely. More concretely, if the attitude matrix $A$ obeys Eq. (1a), then so does the matrix $R\left(\mathbf{b}_{1}, \phi_{b}\right) A R\left(\mathbf{r}_{1}, \phi_{r}\right)$, for any $\phi_{b}$ and $\phi_{r}$, where $R(\mathbf{e}, \phi)$ denotes a rotation by angle $\phi$ about the axis e. This also makes it clear that the attitude is not uniquely determined if either the pair $\left\{\mathbf{b}_{1}, \mathbf{b}_{2}\right\}$ or the pair $\left\{\mathbf{r}_{1}, \mathbf{r}_{2}\right\}$ is collinear.

The earliest algorithm for determining spacecraft attitude from two vector measurements was the TRIAD algorithm ${ }^{12}$, which is simple to implement but does not treat the information in the two observations optimally. Many optimal estimators using two or more measurements have been based on a loss function introduced by Wahba $a^{3.4}$. Shuster showed a simplification of his QUEST algorithm for the two-observation Wahba problem ${ }^{5}$, but reference 6 presented the first explicit closed-form solution. Optimal two-observation algorithms have not found widespread application, since they are significantly slower than TRIAD. In fact, algorithms specifically developed for the two-observation case may require more computations than optimal general purpose algorithms. Recent exceptions are Mortari's optimal EULER-2 algorithm ${ }^{7}$, which approaches TRIAD in speed, and a suboptimal algorithm proposed by Reynolds ${ }^{\mathrm{s}}$. The present paper presents two new algorithms for quaternion estimation from two vector measurements. The first is a very efficient optimal algorithm, which is as fast as the suboptimal TRIAD algorithm. The second produces the same suboptimal estimate as TRIAD, but at reduced computational cost. 
WIHBI'S PROBLE.M

Wahba's problem is to tind the proper orthogonal matrix $t$ that minimizes the loss function

$$
L(A) \equiv \frac{1}{ \pm} \sum_{i} a_{i}\left|\mathbf{b}_{1}-A \mathbf{r}_{1}\right|^{2}
$$

where $\left\{b_{1}\right\}$ is a set of $n$ unit vectors in the spacecraft body frame, $\left\{r_{1}\right\}$ are the corresponding unit vectors in the reference frame, and $\left\{a_{i}\right\}$ are non-negative weights. We use the invariance of the trace under cyclic permutations to rewrite Eq. (3) as

$$
L(A)=\sum_{i} a_{i}-\operatorname{trace}\left(A B^{T}\right)
$$

where

$$
B \equiv \sum_{i} a_{i} \mathbf{b}_{i} \mathbf{r}_{i}^{\top}
$$

Almost all solutions of Wahba's problem are based on the observation that the attitude matrix that maximizes trace $\left(A B^{T}\right)$ minimizes the loss function. The original solutions solved for the attitude matrix $A$ directly, but most practical applications have been based on Davenport's $q$-method ${ }^{2.49}$, which solves for the unit attitude quaternion ${ }^{10,11}$

$$
q=\left[\begin{array}{l}
\mathbf{q} \\
q_{+}
\end{array}\right], \text {where }|q|^{2}=1
$$

There is a $2: 1$ correspondence between the quaternion and the rotation matrix $R(\mathbf{e}, \phi)$ given by

$$
q= \pm\left[\begin{array}{c}
e \sin (\phi / 2) \\
\cos (\phi / 2)
\end{array}\right]
$$

and

$$
A=R(\mathbf{e}, \phi)=\left(q_{\downarrow}^{2}-|\mathbf{q}|^{2}\right) l+2 \mathbf{q} \mathbf{q}^{\mathrm{T}}-2 q_{+}[\mathbf{q} \times]
$$

We will follow Shuster's convention for quaternion products ${ }^{\text {, }}$, writing

$$
p \otimes q=\left[\begin{array}{c}
\mathbf{p} \\
p_{+}
\end{array}\right] \otimes\left[\begin{array}{c}
\mathbf{q} \\
q_{+}
\end{array}\right]=\left[\begin{array}{c}
q_{+} \mathbf{p}+p_{+} \mathbf{q}-\mathbf{p} \times \mathbf{q} \\
p_{+} q_{4}-\mathbf{p} \cdot \mathbf{q}
\end{array}\right]
$$

This differs from the historical convention in the sign of the cross-product, and has the advantage that the order of quaternion multiplication is the same as the order of attitude matrix multiplication. Since the attitude matrix is a homogenous quadratic function of $q$, we can write

$$
\operatorname{tr}\left(A B^{\top}\right)=q^{\top} K q
$$

where $K$ is the symmetric traceless matrix

$$
K=\left[\begin{array}{cc}
B+B^{\mathrm{T}}-I \operatorname{tr} B & \sum_{i} a_{i} \mathbf{b}_{i} \times \mathbf{r}_{i} \\
\sum_{i} a_{i}\left(\mathbf{b}_{i} \times \mathbf{r}_{i}\right)^{\mathrm{T}} & \operatorname{tr} B
\end{array}\right] .
$$

The $q$-method finds the optimal quaternion as the normalized eigenvector of $K$ with the largest eigenvalue, i.e.

$$
K q_{\mathrm{opt}} \equiv \lambda_{\mathrm{max}} q_{\mathrm{upt}}
$$

There is no unique solution if the two largest eigenvalues of $K$ are equal. This is not a failure of the $q$ method; it means that the data aren't sufficient to determine the attitude uniquely.

\section{OPTIMAL QUATERNION ESTIMATION METHOD}

In the two-observation case, it is useful to define the normalized cross products

$$
\mathbf{r}_{3} \equiv\left(\mathbf{r}_{1} \times \mathbf{r}_{2}\right) /\left|\mathbf{r}_{1} \times \mathbf{r}_{2}\right|
$$

and

$$
\mathbf{b}_{3} \equiv\left(\mathbf{b}_{1} \times \mathbf{b}_{2}\right) /\left|\mathbf{b}_{1} \times \mathbf{b}_{2}\right|
$$

We note that $\mathbf{r}_{3}$ or $b_{3}$ is undefined if the reference vectors or the observed vectors, respectively, are collinear. This is the case noted ahove in which there is insufficient information to determine the attitude uniquely. It can be seen from the explicit 
solution $^{6}$, geometrical reasoning ${ }^{7}$, or simply thinking about the loss function of Eq. (3) that the optimal estimate must result in $A \mathbf{r}_{1}$ and $A \mathbf{r}_{2}$ being coplanar with $\mathbf{b}_{1}$ and $\mathbf{b}_{2}$. This means that the optimal rotation maps the cross product vectors as

$$
A_{\mathrm{opt}} \mathbf{r}_{3}=\mathbf{b}_{3} \text {. }
$$

The TRIAD estimate, although not optimal, always obeys this equation; but not all two-measurement estimates do ${ }^{12}$.

The quaternion rotating $\mathbf{r}_{3}$ into $\mathbf{b}_{3}$ with the minimum-angle rotation is, up to an overall sign,

$$
q_{\min } \equiv \frac{1}{\sqrt{2\left(1+\mathbf{b}_{3} \cdot \mathbf{r}_{3}\right)}}\left[\begin{array}{c}
\mathbf{b}_{3} \times \mathbf{r}_{3} \\
1+\mathbf{b}_{3} \cdot \mathbf{r}_{3}
\end{array}\right] \text {. }
$$

The most general rotation that maps $\mathbf{r}_{3}$ into $\mathbf{b}_{3}$ is the minimum rotation preceded by a rotation through an arbitrary angle $\phi_{r}$ about $\mathbf{r}_{3}$ and followed by a rotation through an arbitrary angle $\phi_{b}$ about $\mathbf{b}_{3}$. As observed by Reynolds ${ }^{8}$, this has the quaternion representation

$$
q=\frac{ \pm 1}{\sqrt{2\left(1+\mathbf{b}_{3} \cdot \mathbf{r}_{3}\right)}}\left[\begin{array}{c}
\mathbf{b}_{3} \sin \left(\phi_{b} / 2\right) \\
\cos \left(\phi_{b} / 2\right)
\end{array}\right] \otimes\left[\begin{array}{c}
\mathbf{b}_{3} \times \mathbf{r}_{3} \\
1+\mathbf{b}_{3} \cdot \mathbf{r}_{3}
\end{array}\right] \otimes\left[\begin{array}{c}
\mathbf{r}_{3} \sin \left(\phi_{r} / 2\right) \\
\cos \left(\phi_{r} / 2\right)
\end{array}\right]=q_{\min } \cos (\phi / 2)+q_{180} \sin (\phi / 2),
$$

where $\phi \equiv \phi_{b}+\phi_{r}$ and

$$
q_{180} \equiv \frac{1}{\sqrt{2\left(1+\mathbf{b}_{3} \cdot \mathbf{r}_{3}\right)}}\left[\begin{array}{c}
\mathbf{b}_{3}+\mathbf{r}_{3} \\
0
\end{array}\right]
$$

The quaternion $q_{180}$ rotates the cross product vector $\mathbf{r}_{3}$ into the body frame vector $\mathbf{b}_{3}$ by means of a $180^{\circ}$ rotation about the bisector of these two vectors.

Now we can find the optimal quaternion by finding the angle $\phi$ that minimizes Wahba's loss function. Using Eqs. (4), (10), (16), and the half-angle formulas of trigonometry, we compute

$$
L(A)=a_{1}+a_{2}-\left(1+\mathbf{b}_{3} \cdot \mathbf{r}_{3}\right)^{-1}(\alpha \cos \phi+\beta \sin \phi),
$$

where

$$
q_{\min }^{\mathrm{T}} K q_{\min }=-q_{180}^{\mathrm{T}} K q_{180}=\left(1+\mathbf{b}_{3} \cdot \mathbf{r}_{3}\right)^{-1} \alpha
$$

and

with

$$
q_{\min }^{\mathrm{T}} K q_{180}=q_{180}^{\mathrm{T}} K q_{\min }=\left(1+\mathbf{b}_{3} \cdot \mathbf{r}_{3}\right)^{-1} \beta,
$$

and

$$
\alpha \equiv\left(1+\mathbf{b}_{3} \cdot \mathbf{r}_{3}\right)\left(a_{1} \mathbf{b}_{1} \cdot \mathbf{r}_{1}+a_{2} \mathbf{b}_{2} \cdot \mathbf{r}_{2}\right)+\left(\mathbf{b}_{3} \times \mathbf{r}_{3}\right) \cdot\left(a_{1} \mathbf{b}_{1} \times \mathbf{r}_{1}+a_{2} \mathbf{b}_{2} \times \mathbf{r}_{2}\right)
$$

$$
\beta \equiv\left(\mathbf{b}_{3}+\mathbf{r}_{3}\right) \cdot\left(a_{1} \mathbf{b}_{1} \times \mathbf{r}_{1}+a_{2} \mathbf{b}_{2} \times \mathbf{r}_{2}\right) .
$$

The loss function is minimized by setting $\cos \phi=\alpha / \gamma$ and $\sin \phi=\beta / \gamma$, with

$$
\gamma \equiv \sqrt{\alpha^{2}+\beta^{2}}
$$

which gives

$$
L\left(A_{\mathrm{cpt}}\right)=a_{1}+a_{2}-\left(1+\mathbf{b}_{3} \cdot \mathbf{r}_{3}\right)^{-1} \gamma .
$$

The attitude quaternion estimate requires half-angles. To avoid singularities, we use $\cos (\phi / 2)=\sqrt{\frac{1}{2}(1+\cos \phi)}$ and $\sin (\phi / 2)=\sin \phi / \sqrt{2(1+\cos \phi)}$ when $\cos \phi \geq 0$, and $\sin (\phi / 2)=\sqrt{\frac{1}{2}(1-\cos \phi)}$ and $\cos (\phi / 2)=\sin \phi / \sqrt{2(1-\cos \phi)}$ when $\cos \phi \leq 0$. This gives, finally,

and

$$
q_{\mathrm{upt}}=\frac{1}{2 \sqrt{\gamma(\gamma+\alpha)\left(\mathbf{I}+\mathbf{b}_{3} \cdot \mathbf{r}_{3}\right)}}\left[\begin{array}{c}
(\gamma+\alpha)\left(\mathbf{b}_{3} \times \mathbf{r}_{3}\right)+\beta\left(\mathbf{b}_{3}+\mathbf{r}_{3}\right) \\
(\gamma+\alpha)\left(\mathbf{I}+\mathbf{b}_{3} \cdot \mathbf{r}_{3}\right)
\end{array}\right] \quad \text { for } \alpha \geq 0,
$$

$$
q_{\mathrm{vpt}}=\frac{1}{2 \sqrt{\gamma(\gamma-\alpha)\left(1+\mathbf{b}_{3} \cdot \mathbf{r}_{3}\right)}}\left[\begin{array}{c}
\beta\left(\mathbf{b}_{3} \times \mathbf{r}_{3}\right)+(\gamma-\alpha)\left(\mathbf{b}_{3}+\mathbf{r}_{3}\right) \\
\beta\left(1+\mathbf{b}_{3} \cdot \mathbf{r}_{3}\right)
\end{array}\right] \quad \text { for } \alpha \leq 0 .
$$


The averall sign of the quaternion is irrelevant hecause of the quadratic nature of the attitude representation of Ey. 18 ). This algorithm is very similar to Mortari's ELLER-2 solution to the two-observation Wahba problem", hut it avoids explicit irigonometric function evaluations.

\section{REFERENCE FRAME ROTATIONS}

The ahove expressions for all the components of the optimal quaternion go to the indeterminate expression $0 / 0$ when $\mathbf{b}_{3}=-\mathbf{r}_{3}$. This singular condition can be avoided by solving for the attitude with respect to a reference coordinate frame related to the original frame by $180^{\circ}$ rotations about the $x, y$, or $z$ coordinate axis ${ }^{4.5,13}$. That is, we solve for one of the quaternions

$$
q^{\prime} \equiv q \otimes\left[\begin{array}{l}
\mathbf{e}_{i} \\
0
\end{array}\right]=\left[\begin{array}{l}
\mathbf{q} \\
q_{+}
\end{array}\right] \otimes\left[\begin{array}{l}
\mathbf{e}_{i} \\
0
\end{array}\right]=\left[\begin{array}{c}
q_{+} \mathbf{e}_{i}-\mathbf{q} \times \mathbf{e}_{i} \\
-\mathbf{q} \cdot \mathbf{e}_{i}
\end{array}\right] \quad \text { for } i=1,2,3
$$

where $\mathbf{e}_{i}$ is the unit vector along the $i^{\text {th }}$ coordinate axis. These quaternion products are trivial to implement by merely permuting and changing signs of the quaternion components. For example,

$$
q^{\mathrm{l}}=\left[q_{1}, q_{2}, q_{3}, q_{4}\right]^{\mathrm{T}} \otimes[1,0,0,0]^{\mathrm{T}}=\left[q_{4},-q_{3}, q_{2},-q_{1}\right]^{\mathrm{T}}
$$

The equations for the inverse transformations are the same, since a $180^{\circ}$ rotation in the opposite direction has the same effect. These rotations are also easy to implement on the input data, since a rotation about axis $i$ simply changes the signs of the $j^{\text {th }}$ and $k^{\text {th }}$ columns of $\mathbf{r}_{1}, \mathbf{r}_{2}$, and $\mathbf{r}_{3}$, where $\{i, j, k\}$ is a permutation of $\{1,2,3\}$.

The original QUEST implementation performed sequential rotations one axis at a time, until an acceptable reference coordinate system was found. It is clearly preferable to save computations by choosing a single desirable rotation as early in the computation as possible. Consider the effect on the inner product $\mathbf{b}_{3} \cdot \mathbf{r}_{3}$ of rotating the reference frame about the $i^{\text {th }}$ axis

$$
\left(\mathbf{b}_{3} \cdot \mathbf{r}_{3}\right)_{\text {rotated }}=\left[\left(\mathbf{b}_{3}\right)_{i}\left(\mathbf{r}_{3}\right)_{i}-\left(\mathbf{b}_{3}\right)_{j}\left(\mathbf{r}_{\mathbf{3}}\right)_{j}-\left(\mathbf{b}_{3}\right)_{k}\left(\mathbf{r}_{3}\right)_{k}\right]_{\text {unnotated }}=\left[2\left(\mathbf{b}_{3}\right)_{i}\left(\mathbf{r}_{3}\right)_{i}-\mathbf{b}_{3} \cdot \mathbf{r}_{3}\right]_{\text {unrowied }} .
$$

We can maximize this inner product in the rotated frame by performing no rotation if $\mathbf{b}_{3} \cdot \mathbf{r}_{3}$ is the maximum of $\left\{\left(\mathbf{b}_{3}\right)_{i}\left(\mathbf{r}_{3}\right)_{i},\left(\mathbf{b}_{3}\right)_{j}\left(\mathbf{r}_{3}\right)_{j},\left(\mathbf{b}_{3}\right)_{k}\left(\mathbf{r}_{3}\right)_{k}, \mathbf{b}_{3} \cdot \mathbf{r}_{3}\right\}_{\text {unrouted }}$, while a rotation about the $i^{\text {th }}$ axis is performed if $\left(\mathbf{b}_{3}\right)_{i}\left(\mathbf{r}_{3}\right)_{i}$ is the maximum. This will ensure the largest value for $\mathbf{b}_{3} \cdot \mathbf{r}_{3}$ in the rotated frame. The rotation is easily "undone" by Eq. (27) or its equivalent after the quaternion has been computed.

\section{SLBOPTIMAL QUATERNION ESTIMATION METHOD}

In the limit that the first measurement is far more accurate than the second, Eq. (la) is satisfied exactly. This is often the case of interest, and is the case generally treated by the TRIAD algorithm ${ }^{1.2}$. The quaternion estimate taking $\mathbf{r}_{1}$ into $\mathbf{b}_{1}$ is given, in analogy with Eq. (16), by

$$
q=\frac{ \pm 1}{\sqrt{2\left(1+\mathbf{b}_{1} \cdot \mathbf{r}_{1}\right)}}\left\{\cos (\psi / 2)\left[\begin{array}{c}
\mathbf{b}_{1} \times \mathbf{r}_{1} \\
1+\mathbf{b}_{1} \cdot \mathbf{r}_{1}
\end{array}\right]+\sin (\psi / 2)\left[\begin{array}{c}
\mathbf{b}_{1}+\mathbf{r}_{1} \\
0
\end{array}\right]\right\} \text {. }
$$

We compute the TRIAD-equivalent quaternion by finding the angle $\psi$ that minimizes Wahba's loss function. In parallel with the optimal case, we find

$$
L(A)=a_{2}\left[\mathbf{l}-\left(\mathbf{b}_{1} \cdot \mathbf{b}_{2}\right)\left(\mathbf{r}_{1} \cdot \mathbf{r}_{2}\right)-\left(1+\mathbf{b}_{1} \cdot \mathbf{r}_{1}\right)^{-1}(\mu \cos \psi+v \sin \psi)\right]
$$

where

$$
\mu \equiv\left(1+\mathbf{b}_{1} \cdot \mathbf{r}_{1}\right)\left[\left(\mathbf{b}_{\mathbf{i}} \times \mathbf{b}_{2}\right) \cdot\left(\mathbf{r}_{1} \times \mathbf{r}_{2}\right)\right]-\left[\mathbf{b}_{1} \cdot\left(\mathbf{r}_{1} \times \mathbf{r}_{2}\right)\right]\left[\mathbf{r}_{1} \cdot\left(\mathbf{b}_{1} \times \mathbf{b}_{2}\right)\right]
$$

and

$$
v \equiv\left(\mathbf{b}_{1}+\mathbf{r}_{1}\right) \cdot\left[\left(\mathbf{b}_{1} \times \mathbf{b}_{2}\right) \times\left(\mathbf{r}_{1} \times \mathbf{r}_{2}\right)\right]
$$

The loss function is minimized by setting $\cos \psi=\mu / \rho$ and $\sin \psi=v / \rho$. with

$$
\rho \equiv \sqrt{\mu^{2}+v^{2}}
$$

which gives

$$
L\left(A_{\mathrm{TRIAD}}\right)=a_{2}\left[1-\left(\mathbf{b}_{1} \cdot \mathbf{b}_{2}\right)\left(\mathbf{r}_{1} \cdot \mathbf{r}_{2}\right)-\left(1+\mathbf{b}_{1} \cdot \mathbf{r}_{1}\right)^{-1} \rho\right]
$$


The attitude quaternion estimate is

$$
q_{\mathrm{TR} i \lambda \mathrm{D}}=\frac{1}{2 \sqrt{\rho(\rho+\mu)\left(1+\mathbf{b}_{1} \cdot \mathbf{r}_{1}\right)}}\left[\begin{array}{c}
(\rho+\mu)\left(\mathbf{b}_{1} \times \mathbf{r}_{1}\right)+\nu\left(\mathbf{b}_{1}-\mathbf{r}_{1}\right) \\
(\rho+\mu)\left(1+\mathbf{b}_{1} \cdot \mathbf{r}_{1}\right)
\end{array}\right] \quad \text { for } \mu \geq 0 .
$$

and

$$
q_{\text {TRIAD }}=\frac{1}{2 \sqrt{\rho(\rho-\mu)\left(1+\mathbf{b}_{1} \cdot \mathbf{r}_{1}\right)}}\left[\begin{array}{c}
\nu\left(\mathbf{b}_{1} \times \mathbf{r}_{1}\right)+(\rho-\mu)\left(\mathbf{b}_{1}+\mathbf{r}_{1}\right) \\
v\left(\mathbf{I}+\mathbf{b}_{1} \cdot \mathbf{r}_{1}\right)
\end{array}\right] \quad \text { for } \mu \leq 0
$$

This estimate is less expensive to compute than the optimal estimate, since it is not necessary to normalize the cross products $\mathbf{r}_{1} \times \mathbf{r}_{2}$ and $\mathbf{b}_{1} \times \mathbf{b}_{2}$. However, Eqs. (31) and (32) show that both $\mu$ and $v$ are zero and the estimate is undefined if either of these cross products is zero, indicating collinearity of the reference or body frame vectors. The case of $\mathbf{b}_{1}=-\mathbf{r}_{1}$ is handled by reference frame rotations in the same manner as for the optimal estimate.

\section{TESTS}

MATLAB implementations of these algorithms were coded and tested. The computational speed of the new optimal algorithm is 156 or 158 floating point operations, depending on whether or not a reference frame rotation is required. These are exactly the same number of flops as used by the non-optimal TRIAD algorithm followed by the extraction of a quaternion from the TRIAD attitude matrix. The effort for the optimal algorithm includes the computation of the loss function, which is not provided by TRIAD, however. The suboptimal quaternion estimation algorithm required 107 or 109 flops, without computation of the loss function.

For accuracy tests, we simulate 1000 test cases with uniformly distributed random attitude quaternions. For each attitude we generate two random observation vectors, independently and uniformly distributed on the unit sphere. We use inverse of the attitude quaternion to map the observation vectors to the reference frame. The reference vectors are corrupted by Gaussian random noise with specified standard deviations and then normalized. The optimal estimation algorithm uses the inverse squared measurement standard deviations as the weights in Wahba's loss function.

Two different test scenarios were simulated. The first scenario has measurement standard deviations of 1 arc minute per axis on the first vector and $2^{\circ}$ on the second, which would be appropriate for measurements of a fine digital sun sensor and a triaxial magnetometer, respectively. The attitude estimation errors for this scenario, the rotation angles between true attitude and the estimates, are plotted in Figure 1 as a function of $\left|b_{1} \times b_{2}\right|$. Since the errors are expected to be roughly inversely proportional to $\left|b_{1} \times b_{2}\right|$, the errors have been multiplied by this quantity for plotting, allowing the errors to be plotted on a more-or-less uniform scale for the entire range of angles between the observed vectors ${ }^{6}$. The points for the suboptimal estimation errors and the optimal errors fall on top of each other in Figure 1; they are indistinguishable at the plotting accuracy. This was to be expected with the large the ratio of the measurement weights, $120^{2}$, in this scenario.

The second scenario, shown in Figure 2, used equal measurement standard deviations of $2^{\circ}$ on both, as would be appropriate for measurements of a coarse sun sensor and a triaxial magnetometer. This scenario clearly exhibits differences between the suboptimal optimal estimation errors. This is not surprising, since the difference between the suboptimal TRIAD estimator and the optimal is greatest in the case of equal weights. Interestingly enough, the errors agree very closely when they are greater than $20^{\circ}$, as shown by the points above the diagonal line from $(0,0)$ to $(0.7,14)$ in Figure 2 . These solutions with large errors are not very useful, however. In fact, the figures show that two-observation estimators should not be used for $\left|b_{1} \times b_{2}\right|<0.4$ if it is desired to limit the estimation errors to be less than $10^{\circ}$ in the first scenario or in $20^{\circ}$ the second.

It appears that the optimal estimates have smaller errors than the TRIAD estimates in the second scenario, but this is difficult to quantify by examining Figure 2 . To establish the advantage of the optimal estimator, the probability distribution of $\left|\mathbf{b}_{1} \times \mathbf{b}_{2}\right|$ times the estimation errors in 10,000 simulations of the second scenario are plotted in Figure 3. It is clear from this figure that the optimal estimator offers only marginal gains over the TRIAD estimate. At the $95 \%$ confidence level, $\left|\mathbf{b}_{1} \times \mathbf{b}_{2}\right|$ times the errors of the optimal and suboptimal algorithms are less than $5.3^{\circ}$ and $5.6^{\circ}$, respectively; these scaled errors are less than $6.7^{\circ}$ and $6.9^{\circ}$ at the $99 \%$ confidence level. 


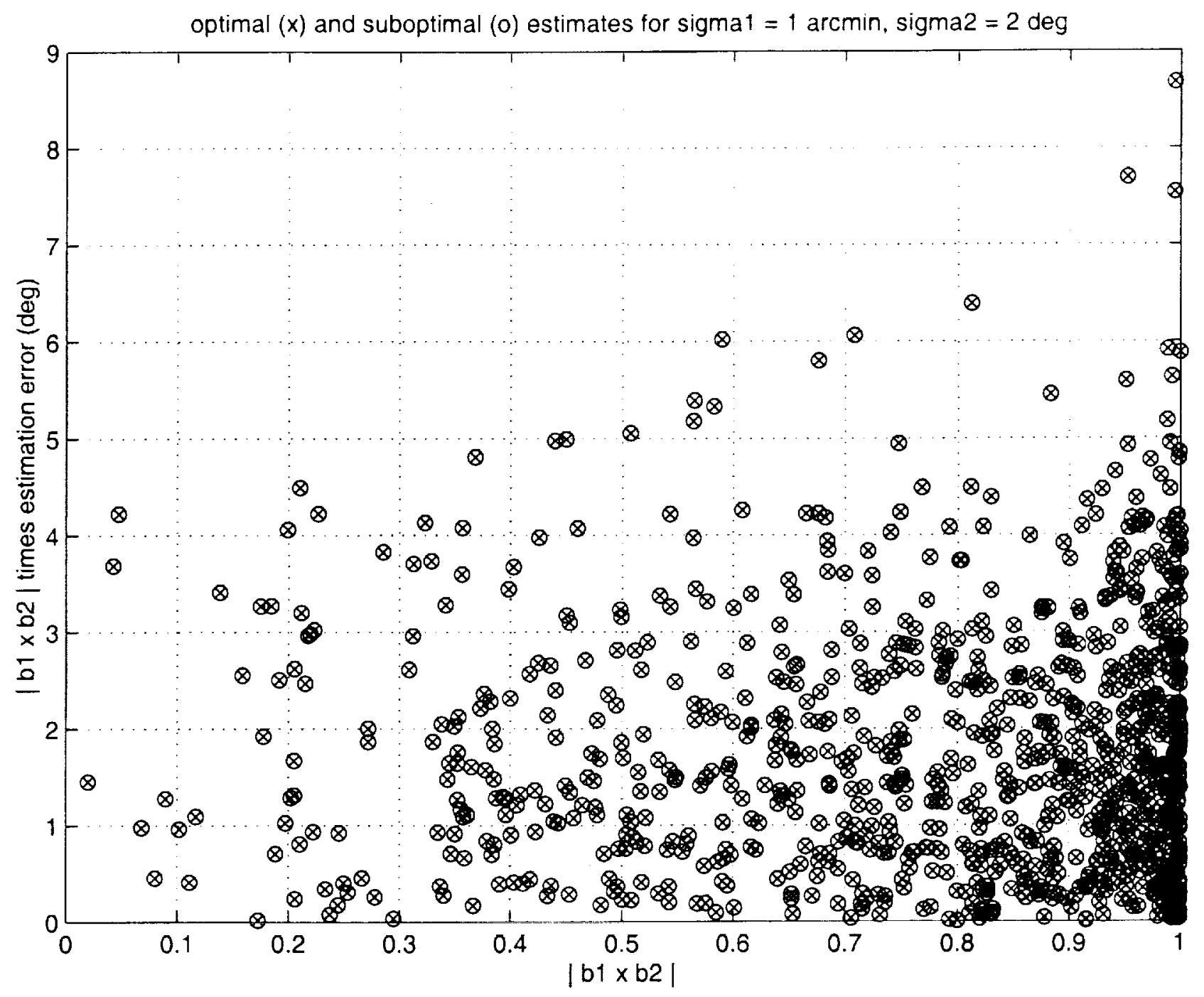

Figure 1. Estimation errors in scenario with unequal measurement errors

\section{CONCLUSIONS}

We have found two new, fast quaternion estimation methods using exactly two vector measurements. These methods are applicable to a variety of problems, including coarse "sun-mag" attitude estimation using the unit vector to the Sun and the Earth's magnetic field vector and precise estimation using unit vectors to stars tracked by two star trackers. The first of the algorithms is optimal in Wahba's sense and is as fast as the TRIAD algorithm, even including computation of Wahba's loss function, which TRIAD does not provide. The second, suboptimal algorithm provides estimates identical to TRIAD with reduced computational effort. The accuracy of these algorithms was examined in simulations of two scenarios, one with equal measurement errors and one with errors differing by two orders of magnitude. These simulations show that the TRIAD estimate is almost as accurate as the optimal estimate in both scenarios. 


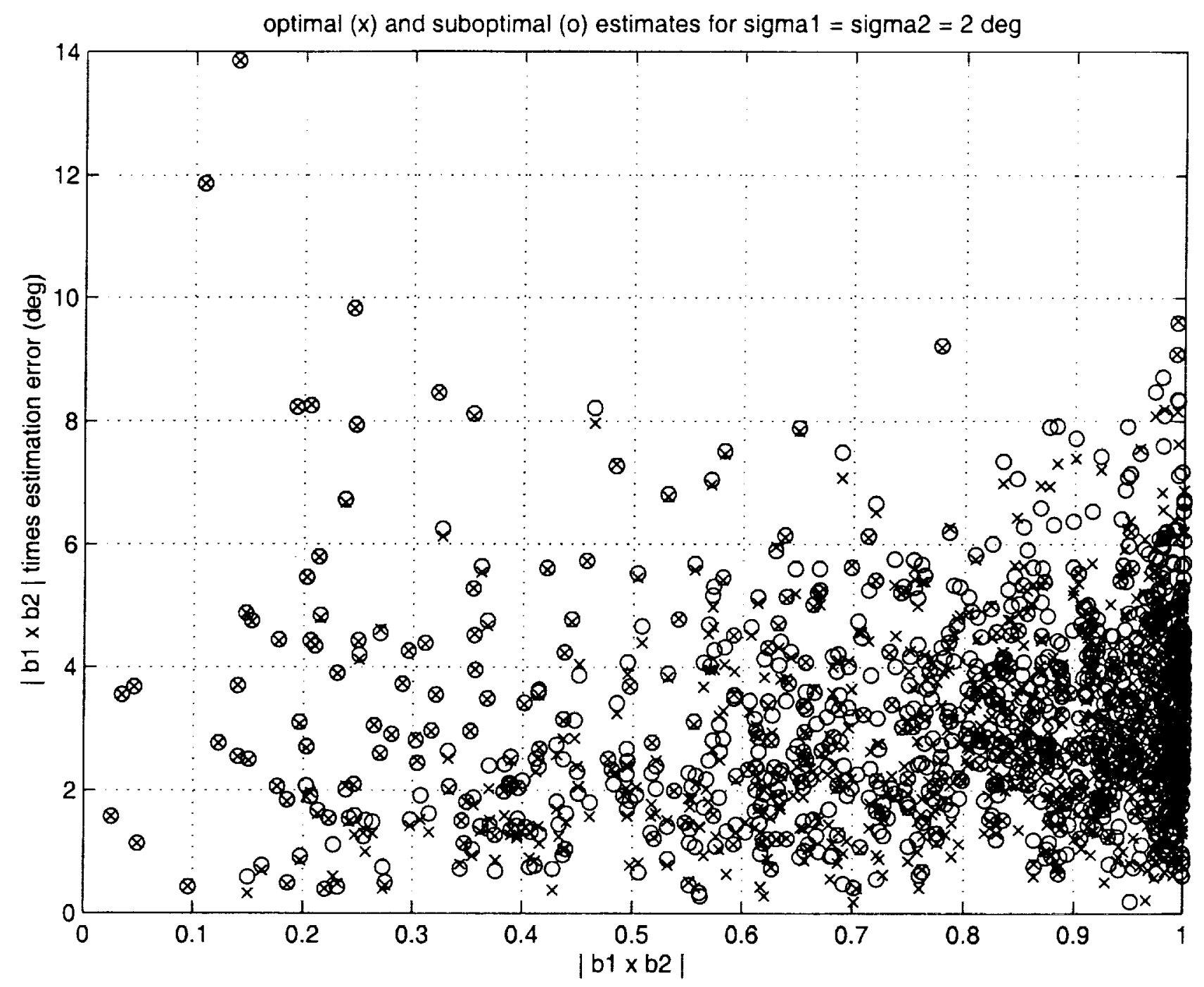

Figure 2. Estimation errors in scenario with equal measurement errors

\section{REFERENCES}

1. Black, Harold D., "A Passive System for Determining the Attitude of a Satellite," AIAA Journal, Vol. 2, No. 7, July 1964, pp. 1350-1351.

2. Lerner, Gerald M., "Three-Axis Attitude Determination," in Spacecraft Attitude Determination and Control, James R. Wertz, ed., D. Reidel, Dordrecht, Holland 1978.

3. Wahba, Grace, "A Least Squares Estimate of Spacecraft Attitude," SIAM Review, Vol. 7, No. 3, July 1965, p. 409.

4. Markley, F. Landis, and Mortari, Daniele, "Quaternion Attitude Estimation Using Vector Observations," Journal of the Astronautical Sciences, Vol. 48, No. 2/3, April-September 2000, pp. 359-380.

5. Shuster, M. D. and S. D. Oh, "Three-Axis Attitude Determination from Vector Observations," Journal of Guidance and Control, Vol. 4, No. 1, January-February 1981. pp. 70-77.

6. Markley, F. Landis, "Attitude Determination Using Vector Observations: A Fast Optimal Matrix Algorithm," Journal of the Astronautical Sciences, Vol. 41, No. 2, April-June 1993, pp. 261-280. 


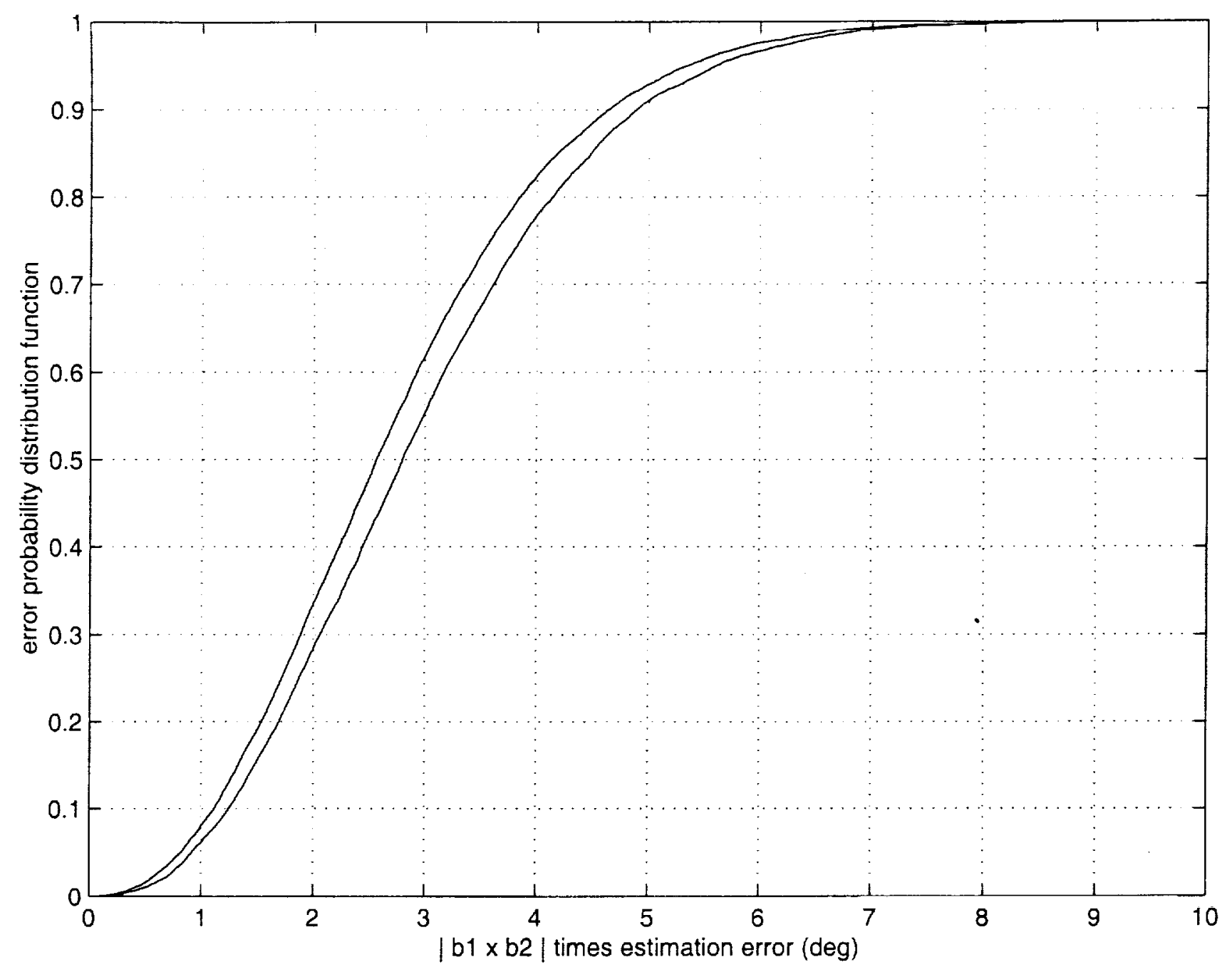

Figure 3. Probability distribution of estimation errors in scenario with equal measurement errors Left curve for optimal estimator, right curve for suboptimal.

7. Mortari, Daniele, "EULER-2 and EULER-n Algorithms for Attitude Determination from Vector Observations," Space Technology, Vol. 16, No. 5/6, 1996, pp. 317-3121.

8. Reynolds, R. G., "Quaternion Parameterization and a Simple Algorithm for Global Attitude Estimation," Journal of Guidance, Control, and Dynamics, Vol. 21, No. 4, July-August 1998, pp. 669-671.

9. Keat, J., "Analysis of Least Squares Attitude Determination Routine DOAOP," Computer Sciences Corporation, CSC/TM-77/6034, February 1977.

10. Markley, F. Landis, "Parameterization of the Attitude," in Spacecraft Attitude Determination and Control, James R. Wertz, ed., D. Reidel, Dordrecht, Holland 1978.

11. Shuster. Malcolm D., "A Survey of Attitude Representations," Journal of the Astronautical Sciences, Vol. 41, No. 4 October-December 1993, pp. 439-517.

12. Markley, F. Landis, "Attitude Determination Using Two Vector Measurements." 1999 Flight Mechanics Symposium, edited by John P. Lynch, NASA Goddard Space Flight Center, Greenbelt, MD, May 18-20, 1999, NASA Conference Publication NASA/CP-19989-209235, pp. 39-52.

13. Shuster, Malcolm D. and Gregory A. Natanson, "Quaternion Computation from a Geometric Point of View," Journal of the Astronautical Sciences, Vol. 41, No. 4, October-December 1993. pp. 545-556. 